\title{
De-noising Method Based on Kernel Adaptive Filtering for Telemetry Vibration Signal of the Vehicle Test
}

\author{
Kejun ZENG \\ PLA 91550 Unit 94, Liaoning Dalian, 116023, China \\ Zengkejun0739@aliyun.com
}

Keywords: Telemetry; Kernel Adaptive Filtering; Vibration Signal; De-noising

\begin{abstract}
A kernel adaptive filtering de-noising method for telemetry signal was proposed. The telemetry vibration signal is often interfered with Gaussian noise and impulse noise at the same time, the tradition de-noising method cannot obtain good de-noising performance. In the vehicle test, the vibration signal has typical non-stationary and non-linear characteristics, for which linear filtering method is failure. Kernel adaptive filtering transforms the input data to high dimension feature space and designs a linear method to solve the nonlinear problem. The proposed method uses kernel adaptive filtering to suppress the noise in the vibration signal of the vehicle test under norm cost function, which can keep effective under non-stationary and non-linear conditions. The simulation and test data processing results show the good performance of the proposed method which can apply in the telemetry vibration signal processing.
\end{abstract}

\section{Introduction}

Telemetry signal is the main basis for the vehicle interior ballistics analysis and detection, in which the vibration signal is the most representative that can directly reflect the response of the vehicle structure state under different incentive conditions, and it processing results can be used to determine the health status of the vehicle structure [1]. The telemetry signal is often interfered with noise during the transmission and reception process by transmitting and receiving equipment and the transmission of the external environment influence. The noise in the signal decrease the average variance estimation precision in time domain analysis, furthermore, serious noise interference can lead to the difficulty of frequency domain analysis and time domain analysis. Therefore, de-noising is an important part in the preprocessing of telemetry vibration signal. The analysis of lots of the experimental data show that the noise in the telemetry signal is often not a simple Gaussian noise, for the high bit error generated during the transmission process also lead to impulse noise. Therefore, the noise in the telemetry signal usually shows the mixed noise form of Gaussian noise and impulse noise [2]. Tradition de-noising method includes frequency domain filtering, median filtering, wavelet de-noising, singular value decomposition (SVD) and empirical mode decomposition (EMD) based on local wave analysis, in which the wavelet threshold de-noising method is most widely used in engineering. In the de-noising methods, frequency domain filtering request signal meets the stationary distribution, median filtering is suitable for impulse noise, the wavelet threshold de-noising and SVD needs to set artificial parameters which is difficult to obtain ideal performance, EMD de-noising takes the high intrinsic mode function (IMF) as the noise which lack of theory basis. Meanwhile, all kinds of the de-noising methods meet the difficulty that to find the best combination points of noise suppression and signal loss of detail information. For the mixed noise form de-noising, the single de-noising method has poor performance, and in vehicle test data detection proves that the telemetry signals often appear non-stationary characteristics [3]. Kernel adaptive filtering provides a new method for signal de-noising, which can map the input signal to the high dimension feature space to obtain good filtering performance under nonlinear condition. By using the kernel adaptive filtering method, a new method of telemetry vibration signal de-noising was proposed, which can solve the de-noising problem for mixed noise form of Gaussian noise and impulse noise and has good performance for non-stationary telemetry vibration de-noising. The computer simulation and experimental data processing results show the 
effectiveness of the proposed method.

\section{Basic Principles of Kernel Adaptive Filtering}

Kernel method provides a robust nonlinear parameter modeling method [4]. So far, the kernel method has been successfully applied to support vector machine (SVM), kernel principal component analysis (KPCA) and Fisher discriminant analysis. The basic idea of kernel methods is that mapping the input data to a high dimension feature space by a reproducing kernel, such that the inner product in the feature space can be more efficient computed by kernel evaluation. In the feature space, the non-linear problem can be solved by linear filtering method if the algorithm can be expressed as inner product form, which can avoid the complex computation process. The mapping of the input signal and high dimension feature space is shown in Fig.1. Where $\varphi$ is the feature mapping and $\varphi(\boldsymbol{u})$ is the feature vector in the feature space, thus the inner product can be expressed by kernel as follow

$$
\varphi(\boldsymbol{u})^{T} \varphi(\boldsymbol{u})=k\left(\boldsymbol{u}, \boldsymbol{u}^{\prime}\right)
$$

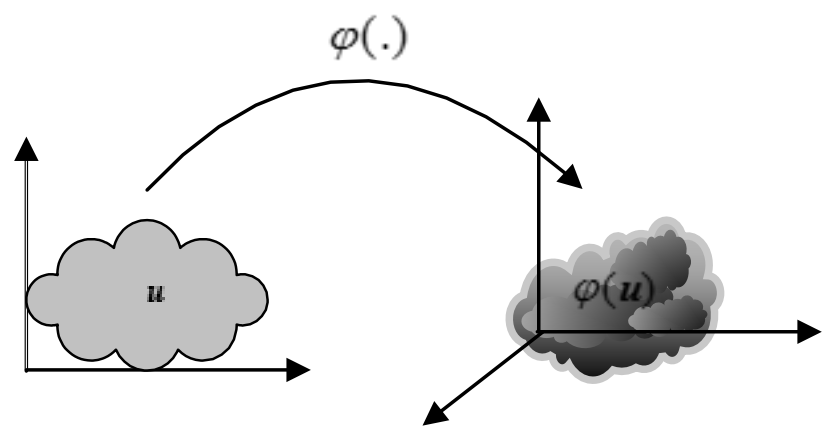

Fig.1 Nonlinear mapping from input space to feature space

The inner product space and reproducing kernel Hilbert space (RKHS) space is essentially the same, and the most commonly used method is the development of kernel filters in RKHS, which can implement the linear adaptive filtering by the linear structure of RKHS. Compared with the neural network, the kernel adaptive filter has universal approximation ability and convex optimization feature, and has no local minimum. Kernel adaptive filtering is a memory intensive operation and the computational complexity with dimension exponential growth. However, under the limited input data, kernel adaptive filtering method can not only complete data processing off-line, also can solve the problem of learning online.

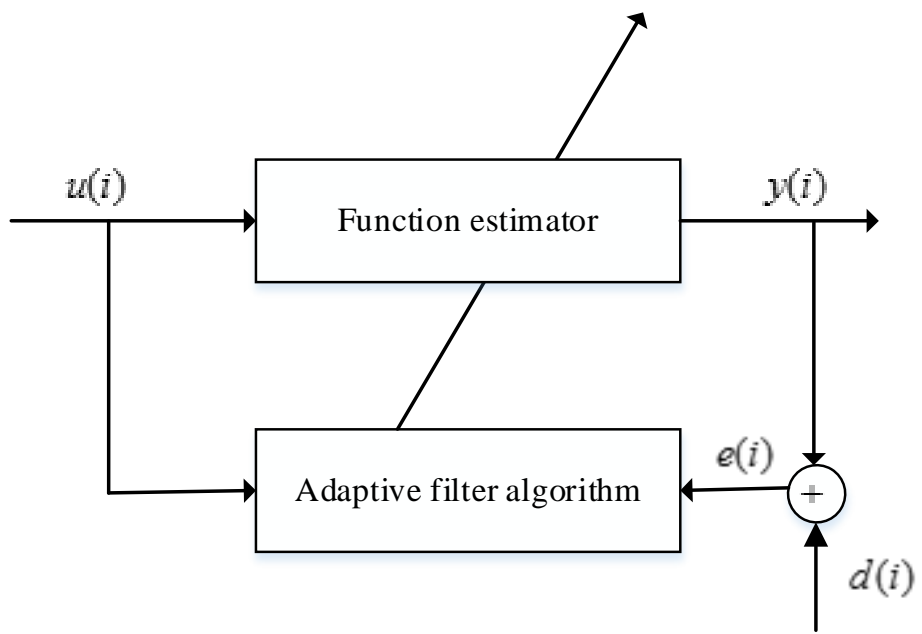

Fig.2 Basic principle of LMS adaptive filter

In the traditional adaptive filtering algorithm, LMS algorithm is robust, simple structure and the most commonly used, if the problem to be solved is linear problems, LMS usually can get the 
desired convergence performance. Fig.2 shows the typical LMS filtering block diagram [5]. Let the input-output sample data is $\{\boldsymbol{u}(1), d(1)\},\{\boldsymbol{u}(2), d(2)\}, \cdots,\{\boldsymbol{u}(N), d(N)\}$, there has continuous input and output mapping relations between continuous samples $f: U \rightarrow R$. As long as the filtering algorithm is fit to the mapping relationship, the LMS adaptive filter can complete the fitting, identification and classification etc.

LMS algorithm is usually to minimize the empirical risk objective function to achieve the optimal function of the parameters of the estimator, which is to obtain the optimal filter weights, the empirical risk cost function is

$$
J(\boldsymbol{w})=\frac{1}{2} \sum_{i=1}^{N}\left[d(i)-\boldsymbol{w}^{T} \boldsymbol{u}(i)\right]^{2}
$$

According to the stochastic gradient descent algorithm, the LMS can update the weight coefficient using the instantaneous gradient as follow

$$
\frac{\partial J(\boldsymbol{w})}{\partial \boldsymbol{w}}=\sum_{i=1}^{N} \boldsymbol{u}(i)\left[d(i)-\boldsymbol{w}^{T} \boldsymbol{u}(i)\right]
$$

Thus the instantaneous gradient at iterative times $i$ is

$$
\nabla J(\boldsymbol{w})=\boldsymbol{u}(i)\left[d(i)-\boldsymbol{w}(i-1)^{T} \boldsymbol{u}(i)\right]
$$

According to the steepest descent algorithm, the LMS algorithm can be expressed as follow

$$
\boldsymbol{w}(i)=\boldsymbol{w}(i-1)+\mu \boldsymbol{u}(i)\left[d(i)-\boldsymbol{w}(i-1)^{T} \boldsymbol{u}(i)\right]
$$

Where $\mu$ is the study step size which controls the convergence rate and the convergence precision of LMS adaptive filtering. If the instantaneous error is defined as follow

$$
e(i)=d(i)-w(i-1)^{T} \boldsymbol{u}(i)
$$

Then the update formula of the LMS algorithm can be written as [6]

$$
\boldsymbol{w}(i)=\boldsymbol{w}(i-1)+\mu e(i) \boldsymbol{u}(i)
$$

LMS algorithm assumes that the filter is a linear finite impulse response filter, if the mapping relationship between the input and output samples is highly non-linear, and then the performance of LMS algorithm will be drastically decreased. According to the principle of kernel adaptive filtering, if the samples can be mapped to high dimension feature space, the iterative algorithm can implement as linear problem. Let the mapping relationship is $\boldsymbol{u}(i) \rightarrow \varphi(\boldsymbol{u}(i))$, for the sake of simplicity, let $\varphi(i)=\varphi(\boldsymbol{u}(i))$, thus in the high dimensional space, a new input and output sample sequence is obtained as $\{\varphi(i), d(i)\}$. The LMS algorithm can be used in the high dimensional feature space as follow

$$
\begin{aligned}
& e(i)=d(i)-\omega(i-1)^{T} \varphi(i) \\
& \omega(i)=\omega(i-1)+\mu e(i) \varphi(i)
\end{aligned}
$$

However, the dimension of $\varphi$ in the feature space is too high and implicit expression. Therefore, an alternative method is needed to complete the calculation, and the iterative process of the iterative Eq.9 can be obtained the follows conclusion.

$$
\begin{aligned}
\omega(i) & =\omega(i-1)+\mu e(i) \varphi(i) \\
& =[\omega(i-2)+\mu e(i-1) \varphi(i-1)]+\mu e(i) \varphi(i) \\
& =\omega(i-2)+\mu[e(i-1) \varphi(i-1)+e(i) \varphi(i)] \\
& =\cdots \\
& =\omega(0)+\mu \sum_{j=1}^{N} e(j) \varphi(j) \\
& =\mu \sum_{j=1}^{N} e(j) \varphi(j)
\end{aligned}
$$


In Eq.10, it is assumed that $\omega(0)=0$, which can meet most of the application conditions. If the system get a new input, the output of the system can be expressed as inner product form as follow.

$$
\begin{gathered}
\boldsymbol{\omega}(i) \boldsymbol{\varphi}\left(u^{\prime}\right)=\left[\mu \sum_{j=1}^{N} e(j) \varphi(j)^{T}\right] \boldsymbol{\varphi}\left(u^{\prime}\right) \\
=\mu \sum_{j=1}^{N} e(j)\left[\boldsymbol{\varphi}(j)^{T} \boldsymbol{\varphi}\left(u^{\prime}\right)\right]
\end{gathered}
$$

In this way, the output of the filter can be evaluated very efficiently by using the kernel technique in the input space.

$$
\omega(i) \varphi\left(u^{\prime}\right)=\mu \sum_{j=1}^{N} e(j) k\left(\varphi(j)^{T} \varphi\left(u^{\prime}\right)\right)
$$

In kernel adaptive filtering, it is no longer involved to update the weights, the filtering operator is completed by all previous estimate error and kernel evaluation function. Kernel adaptive filtering algorithm only requires the inner product operation. The algorithm can save a lot of time, but regardless of the LMS algorithm in the input space and the feature space is essentially the same.

Let $f_{i}$ is the nonlinear mapping of the input and output, the following sequential learning rules can be formed for the kernel adaptive filtering algorithm.

$$
\left\{\begin{array}{c}
f_{i-1}=\mu \sum_{j=1}^{i-1} e(j) k(\boldsymbol{u}(j), .) \\
f_{i-1}(\boldsymbol{u}(i))=\mu \sum_{j=1}^{i=1} e(j) k(\boldsymbol{u}(j), \boldsymbol{u}(i)) \\
e(i)=d(i)-f_{i-1}(\boldsymbol{u}(i)) \\
f_{i}=f_{i-1}+\mu e(i) k(\boldsymbol{u}(i), .)
\end{array}\right.
$$

Eq.13 called kernel LMS (KLMS) algorithm, which is the LMS algorithm in the RHKS. KLMS assigns a new kernel unit to the new training data, and the center of the new kernel unit is the input data $\boldsymbol{u}(i)$, the kernel coefficient is $\mu e(i)$. In the training process, the coefficient and the center are stored in the memory. Fig.3 shows the KLMS algorithm structure. Where $\boldsymbol{a}(i)$ is the coefficient vector at the iterative times $i$ and $\boldsymbol{a}_{j}(i)$ is the $j$-th component of $\boldsymbol{a}(i)$. The system output for the input $\boldsymbol{u}$ can be given by

$$
f(\boldsymbol{u})=\mu \sum_{j=1}^{i} e(j) k(\boldsymbol{u}(j), \boldsymbol{u})
$$

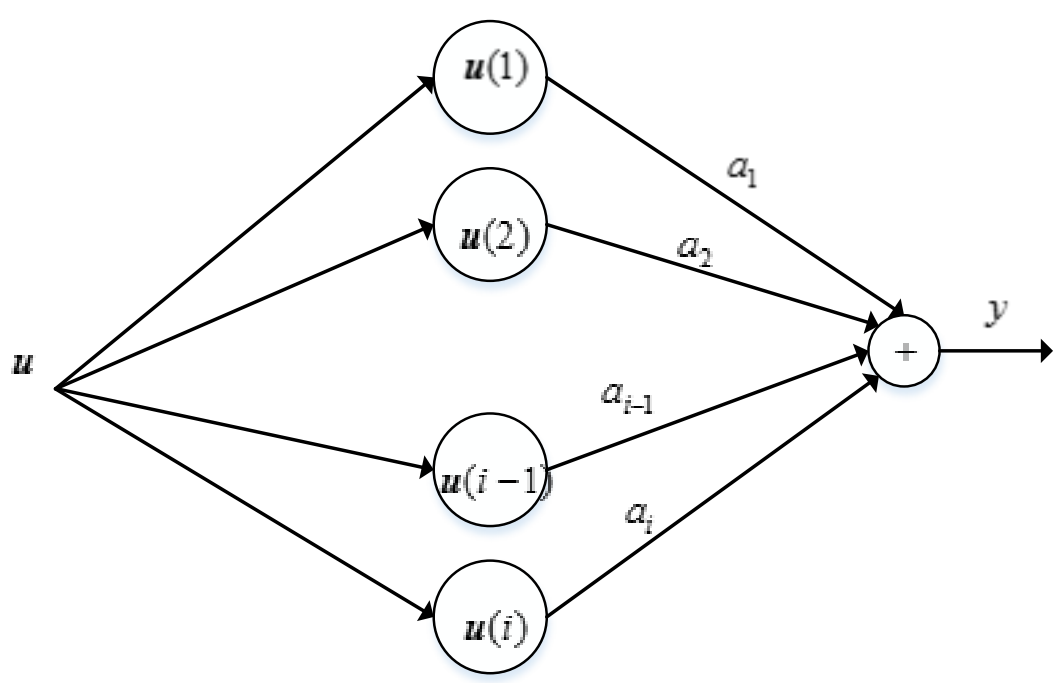

Fig.3 The network topology of KLMS at iterative times $i$ 


\section{De-noising Based on KLMS}

Kernel adaptive filtering is applied to non-linear mapping, which has a unique advantage in the processing of telemetry environment parameters. Telemetry environmental parameters de-noising can take the collected data as the time sequence, which is the sequential learning sample, to realize parametric fitting to complete de-noising by using kernel adaptive filtering. Let telemetry vibration signal is

$$
x(t)=\tilde{x}(t)+v(t)
$$

Where $x(t)$ is the observed signal, $\tilde{x}(t)$ is the useful signal and $v(t)$ is the noise which commonly is the mixed noise of Gaussian noise and impulse noise. Let the cost function is

$$
J(w)=\frac{1}{2}[y(t)-x(t)]^{2}
$$

Where $y(t)$ is the output of the filter. Based on the cost function Eq.16, the vibration signal can be de-noising by KLMS, where the kernel function is selected as Gaussian kernel as follow

$$
k\left(\boldsymbol{u}, \boldsymbol{u}^{\prime}\right)=\exp \left[-\alpha\left\|\boldsymbol{u}-\boldsymbol{u}^{\prime}\right\|^{2}\right]
$$

The simulation frequency hopping signal is used to analyze the performance of the de-noising method, which has typical non-stationary characteristic. The simulation telemetry vibration signal has the practical application background, that is, when the vehicle structure is abnormal, the frequency of the acquisition signal is changed. The sample frequency of the simulation signal is $f_{s}=2048 \mathrm{~Hz}$, the center frequency of two sine signal are $f_{1}=5 \mathrm{~Hz}$ and $f_{2}=25 \mathrm{~Hz}$ respectively. Let the SNR=3dB. The waveforms before de-noising by KLMS are shown in Fig.4 and Fig.5 respectively. Fig.5 shows KLMS has good de-noising performance for the simulation signal.

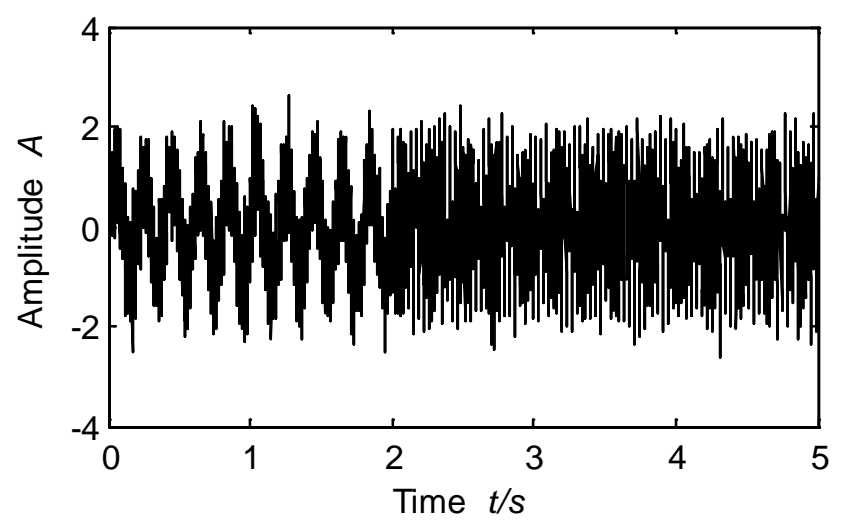

Fig.4 Simulation frequencey hopping signal with noise

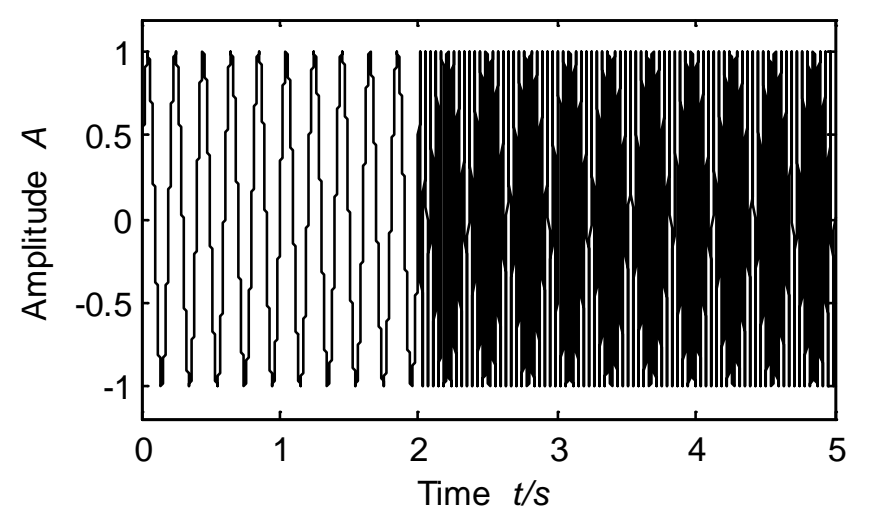

Fig.5 Simulation frequencey hopping signal after de-noising

To further illustrate the practicality of the algorithm, high frequency vibration signal collected by a vehicle test is used for processing, which the sample frequency is $5 \mathrm{kHz}$ and waveform and frequency spectrum is shown in Fig.6. Fig.7 shows the de-noising result of KLMS. The results show that KLMS can suppress the noise in the vibration signal effectively. 

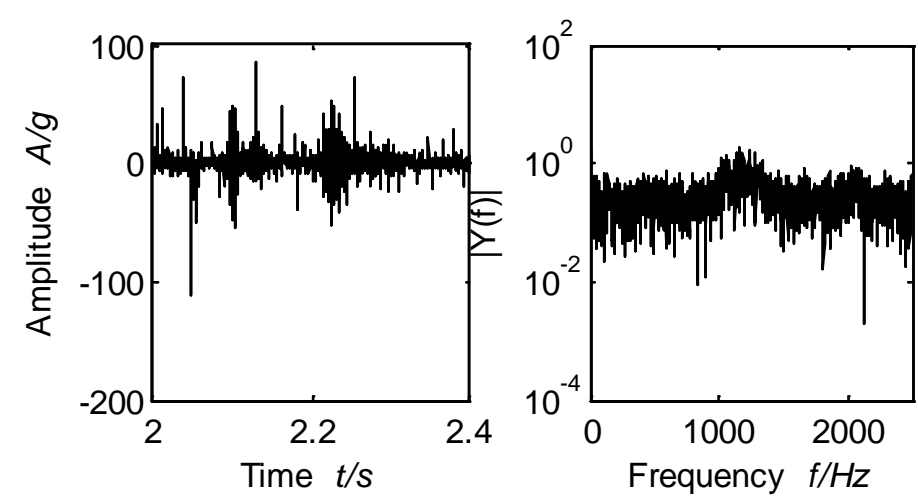

Fig.6 High frequency vibration signal and its frequency spectrum
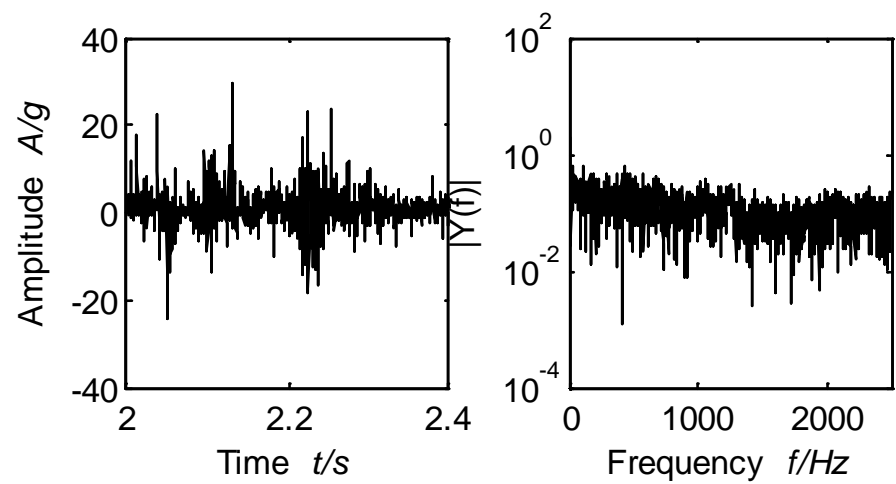

Fig.7 High frequency vibration signal after de-noising

\section{Conclusion}

This work proposed a de-noising method for telemetry signal based on kernel adaptive filtering. For the telemetry environmental parameters in the vehicle test is often interference with mixed noise, the proposed method can obtain good de-noising performance under non-stationary and non-linear condition, which has practical application significance in the telemetry vibration signal de-noising processing. The effectiveness of the proposed method is proved by the simulation signal and the measured signal processing results.

\section{References}

[1] LIU X., LIANG H., XUAN Z.W. Telemetry vibration signal anomaly detection method based on fuzzy entropy of wavelet modulus maxima [J]. Journal of Vibration and Shock, 2016, 35(9): 147-151+158.

[2] LIU X., XUAN Z. W., LIANG H. Telemetry vibration signal abnormality detection method based on multi-scale spectral kurtosis map [J]. Journal of Projectiles, Rockets, Missiles and Guidance, 2015, 35(5): 187-190.

[3] Antoni J., Randall R. B. The spectral kurtosis: a useful tool for characterizing non-stationary signals [J]. Mechanical Systems and Signal Processing, 2006, 20(2): 282-307.

[4] ZHAO J., LIAO X. F., WANG S. Y. Kernel least mean square with single feedback [J]. IEEE Signal Processing Letter, 2015, 22(7): 953-957.

[5] LIU J. C., ZHAO H. Z., QUAN H. D., et al. Iteration based variable step size LMS algorithm and its performance analysis [J]. Journal of Electronics \& Information Technology, 37(7): 1674-1680.

[6] Kwong R. H., Johnston E. W. A variable step size LMS algorithm [J]. IEEE Transactions on Signal Processing, 1992, 40(7): 1633-1642. 